\title{
Negative Gravitational Mass: An Ideal Solution for Cosmology
}

\author{
Stéphane Le Corre \\ Ecole Polytechnique Fédérale de Lausanne, Lausanne, Switzerland \\ Email: stephane.lecorre@epfl.ch
}

How to cite this paper: Le Corre, S. (2020) Negative Gravitational Mass: An Ideal Solution for Cosmology. Open Access Library Journal, 7: e6070.

https://doi.org/10.4236/oalib.1106070

Received: January 13, 2020

Accepted: February 7, 2020

Published: February 10, 2020

Copyright (C) 2020 by author(s) and Open Access Library Inc.

This work is licensed under the Creative Commons Attribution International License (CC BY 4.0).

http://creativecommons.org/licenses/by/4.0/

\section{(c) (i) Open Access}

\begin{abstract}
This article provides a physical solution to cosmic inflation, the onset of the radiation epoch and the reheating epoch. It does not require dark energy thanks to a zero cosmological constant. Cosmological inflation is a mathematical solution considered necessary for the coherence of cosmological models. There are several versions of it, but there is currently no physical basis to explain it. We show that the existence of negative heavy masses (but always positive inert masses) makes it possible to explain it and also makes it possible to justify many other phenomena. Experiments are underway to test the hypothesis of negative heavy masses. The first results should be obtained within 1 or 2 years. In this solution, inflation occurs at the onset of the gravitational interaction much earlier than in the commonly accepted chronology. Gravitation gives rise to a negative pressure primordial fluid verifying the inflation conditions known as "slow roll". It leads to regularity in matter density and temperature of the CMB. The structures of filaments, walls and large cosmic voids (and even superclusters) can be explained as a consequence of the influence of this matter of negative gravitational mass.
\end{abstract}

\section{Subject Areas}

Modern Physics

\section{Keywords}

Negative Gravitational Mass, Cosmological Inflation, Dark Energy, Cosmological Constant, Expansion, Radiation Epoch, Reheating Epoch, Filaments, Walls, Superclusters

\section{Introduction}

The main idea developed in this article to explain the cosmological inflation of 
the $1^{\text {st }}$ time of the Universe is the hypothesis of the existence of negative heavy masses. This hypothesis is being tested by several experiments (notably at CERN). If their discovery is confirmed, it will certainly be one of the most beautiful discoveries (at least equal to gravitational waves). It should be noted at once that the solution presented here differs from other studies on negative masses (for example [1]) by the negativity of the only gravitational mass (the inert mass remaining always positive) which leads to the attractiveness of masses of the same sign (in particular negative masses between them) and to the repulsion of masses of opposite sign. In article [2], we show that the existence of negative heavy masses is not only consistent with General Relativity, but they are even necessary from a theoretical point of view like the antiparticles predicted by $\mathrm{Di}$ rac. The inert mass must also be always positive, which leads to various consequences which can be the subject of experience, in particular on the principle of equality of heavy and inert masses. The two main consequences that we will use in the following are that the gravitational interaction between masses of opposite sign is repulsive (and attractive between masses of the same sign) and that antiparticles necessarily have negative heavy masses. This will allow us to establish a scenario to explain the cosmological inflation of the $1^{\text {st }}$ time of the Universe. In this solution, there will be no dark energy at this epoch of the Universe and the cosmological constant will be zero. I remind you that in this theoretical framework, the other facet of dark energy (necessary to explain, this time, the acceleration of expansion to later ages) and which occurs much later than the epoch treated in this article could also be explained by the negative gravitational mass [3] but by a physical process different from what will be approached here $\left(2^{\text {nd }}\right.$ component of the gravitational field of the general relativity of the negative heavy masses). We will give a physical explanation for the "slow roll" hypothesis expected by theories of cosmological inflation. This inflation is achieved by the symmetry breaking giving rise to the gravitational interaction, therefore earlier than in current theories. Epochs of radiation and reheating are naturally induced at the end of inflation. This solution naturally defines initial conditions to achieve regularity in matter density and in temperature of the CMB. And finally, filaments, walls and superclusters would be the last vestiges of the influence of antimatter, this matter of negative heavy mass. Smaller-scale structures (clusters, galaxies, etc.) are said to be forged by the attractive gravitation of matter specific to each universe.

\section{Elements of Our Solution}

\subsection{Symmetry Breaking of the Gravitational Interaction (Inflation Epoch)}

Our hypothesis considers the existence of negative gravitational mass. As for the positive gravitational masses, they are formed at the time of the appearance of the gravitational interaction which occurs during the $1^{\text {st }}$ symmetry breaking separating the gravitation from the other interactions still unified. Pairs of par- 
ticles composed of a positive heavy mass and a negative heavy mass (its antiparticle) are created such that their sum is zero in terms of heavy mass. The inert mass is always positive. Contrary to current theories of inflation [4], in our solution inflation will therefore have to occur for $t<10^{-43} \mathrm{~s}$. The calculation will verify it.

\subsection{Pair $(m>0$ and $m<0)$ of Repulsive Gravitation (Expansion, Inflation)}

As indicated in the article [3], the repulsive gravitational interaction is realized only between positive and negative heavy masses (contrary to theories supposing the existence of negative masses, for which negative masses also repel each other, [1]). Each of the masses of the pair repel each other with extreme intensity depending on their initial distance. We will henceforth denote $d_{\text {intra }}$ the typical distance between the two positive and negative masses within the pair. We will see that we obtain a characteristic distance $d_{\text {intra }} \sim 10^{-36.4} \mathrm{~m}$ within the pair (at the end of the calculation we will comment on the validity of these values which can rightly make us skeptical). The calculation then gives a negative gravitational pressure of the order of $p \sim-10^{123.8} \mathrm{~N} \cdot \mathrm{m}^{-2}$. In other words, each pair of particles literally explode upon their creation. This solves a first fundamental problem in modern cosmology, the expansion of the Universe. Indeed, while gravitation, according to our current knowledge is only attractive, no known energy source explains this expansionist dynamic of the Universe (regardless of the problem of inflation). And as for the existence of negative heavy masses, this solution (by this initial repulsive impulse) makes this expansion necessary.

\subsection{Gravitational Pressure ("Negative Pressure Matter") and Principle of Our Calculation}

A priori, the primordial cloud resulting from the symmetry breaking giving rise to gravitation is potentially subject to the attractive forces between masses of the same sign and to the repulsive forces between masses of opposite sign. But in our solution, the gravitational attraction is neutralized by the existence of negative heavy mass and by the creation of particle-antiparticle pairs. The heavy mass of the pair is zero because its two masses are opposite heavy masses. Gravitation is therefore immense within the pair and very quickly zero outside the pair. The particle cloud resulting from the symmetry breaking therefore does not undergo the gravitational attraction of the whole cloud as soon as we are at the scale of a few pairs. The primordial impulse (within the pair) will not be thwarted by the gravitational attractiveness of the cloud (whatever its size and inert mass). We can therefore consider (at the time of the symmetry breaking) that only the gravitational force within the pair of opposite masses intervenes in the internal energy of the fluid. The regularity in the fine alternation of the positive and negative masses of this fluid (of the order of $d_{i n t r a}$ ) neutralizes the gravitational interaction beyond the pair. It is the equivalent of the screening effect in electromagnetism. 
In order to obtain an order of magnitude of the pressure exerted by gravitation, we will resume the expression of the gravitational force exerted by $M_{+}$the positive heavy mass on $M_{-}$the negative heavy mass:

$$
\overrightarrow{F_{+/-}} \sim \frac{-G M_{+} M_{-}}{d_{\text {intra }}^{2}} \overrightarrow{u_{+\rightarrow-}}
$$

As $M_{+}=-M_{-}=M$, with $M$ the inert mass always positive, we have:

$$
\overrightarrow{F_{+/-}} \sim \frac{G M^{2}}{d_{\text {intra }}^{2}} \overrightarrow{u_{+\rightarrow-}}
$$

This force is centrifugal (it separates the 2 particles from the pair), the set of pairs of particles created by the symmetry breaking expands this cloud. This primordial impulse therefore generates negative pressure within this cloud. We can express it differently. In this solution, the internal energy of the cloud is due to its gravitational force. However conventionally, the pressure is strictly positive when it is necessary to provide energy to decrease the volume. In our case, we need to remove the gravitational energy from the pair to avoid the increase in volume (and therefore to "decrease" the volume) which justifies the negative pressure.

According to the definition of the pressure, we will obtain the pressure within this primordial cloud by the sum of all the forces exerted on a surface $S$. We work in absolute value on the force but as explained previously this pressure is negative:

$$
p \sim-\varepsilon\left(\sum_{\{M\} \subset S}\left\|\overrightarrow{F_{+/-}}\right\|\right) / S
$$

We have introduced the parameter $\varepsilon$ in order to simplify the calculation. It considers the fact that the forces $\overline{F_{+/-}}$are not necessarily perpendicular to the oriented surface $\vec{S}$. We will therefore have $\varepsilon \leq 1$. This parameter will neither modify the principles of our calculation nor the orders of magnitude obtained. Consequently, we will perform all the calculations with $\varepsilon=1$ and re-introduce it at the end to observe its effect on the results. We can expect to have $\varepsilon>1 / 4$. Therefore, we will give the results for the value $\varepsilon=1 / 4$ which will allow us to note that our solution is stable compared to this definition of the pressure. We could even take smaller values for $\varepsilon$.

In order to obtain an order of magnitude, we will consider a unit surface ( $S=1 \mathrm{~m}^{2}$ ), and we will count the number of particles which are on this surface ( $\{M\} \subset S$ ). Each particle will exert the previous force on the surface ("centrifugal" force compared to this surface for all these particles).

If we denote $d_{\text {inter }}$ the characteristic distance between the pairs, we can expect that the mean distance between particles is a value between $d_{\text {inter }}$ and $d_{\text {intra }}$. For the moment, we are assuming that $d_{\text {inter }}$ is of the same order of magnitude as $d_{\text {intra }}$. We will see later that this assumption will allow us on the one hand to obtain inflation (with $d_{\text {inter }} \sim d_{\text {intra }}$ ) and on the other hand will allow to justify the hypothesis called "slow roll". This allows us to write that the number 
of particles on a unit surface is $\sum_{\{M\} \in\{S=1\}} 1 \sim d_{\text {intra }}^{-2}$. The pressure is then obtained by the formula:

$$
p \sim-\frac{G M^{2}}{d_{\text {intra }}^{4}}
$$

Given the extremely high temperatures at these very early ages of our Universe, we will hypothesize that the kinetic energy of these particles is essentially due to thermal energy and that their speed is close to $c$ the speed of light. This equation will be used at the time of the symmetry breaking giving rise to gravitation (the other interactions are still unified, drowned under the high energy levels of this epoch). We can then write (with $k_{B}$ the Boltzmann constant and $T$ the temperature):

$$
M c^{2}=3 k_{B} T \Rightarrow M=\frac{3 k_{B} T}{c^{2}}
$$

Note: The value of the inert mass of the preceding formula will be the same as that of the gravitational mass because, as indicated in article [2], the principle of equivalence is respected when the objects are not mixtures of particles-antiparticles and this is the mass of the particle (and not of the cloud). We can expect, at this stage of creation of the 1stparticles, that they are pure. They are certainly quarks whose all characteristics except their gravitational mass are still masked by the high energy levels, the other breaking symmetries not having yet occurred.

We can also express $d_{\text {intra }}$ as a function of $\rho_{M}$ the density of matter. As we said before, the average distance between particles will be around $d_{\text {intra }}$. The Universe at this time is made up of a cloud of particles of inert mass $M$ (always positive) spaced from $d_{\text {intra }}$ which allows us to write (the number of particles in a unit volume being $\sum_{\{M\} \subset\{V=1\}} 1 \sim d_{\text {intra }}^{-3}$ ):

$$
\rho_{M}=\left(\sum_{\{M\} \subset V} M\right) / V=M d_{\text {intra }}^{-3} \Rightarrow d_{\text {intra }}=\left(\frac{M}{\rho_{M}}\right)^{1 / 3}
$$

Thanks to this modeling, we now have formulas which only depend on the temperature and the density of matter $\rho_{M}$. However, these two parameters evolve according to known relations which only depend on the parameter $a(t)$ the scale factor of the equations of general relativity of cosmological models. We thus have a modeling entirely dependent on this single parameter $a(t)$.

We will use three other relations of general relativity, one giving the evolution of the temperature and the other two giving the evolution of the density of matter according to the scale factor $a(t)$ and the type of dominant energy:

$$
\frac{T_{t_{1}}}{T_{t_{2}}}=\left(\frac{a\left(t_{1}\right)}{a\left(t_{2}\right)}\right)^{-1}
$$

For a universe dominated by matter:

$$
\frac{\rho_{t_{1}}}{\rho_{t_{2}}}=\left(\frac{a\left(t_{1}\right)}{a\left(t_{2}\right)}\right)^{-3}
$$


For a universe dominated by radiation:

$$
\frac{\rho_{t_{1}}}{\rho_{t_{2}}}=\left(\frac{a\left(t_{1}\right)}{a\left(t_{2}\right)}\right)^{-4}
$$

\section{Inflation, Multiple Childbirth Including Our Universe}

\subsection{The Universe from Now to Inflation}

We now have all the elements to go back in time to inflation. We will note the following initial conditions:

$t_{0}$ the current time. It is 13.7 billion years, i.e. $t_{0} \sim 10^{17.6} \mathrm{~s}$.

$\rho_{0}$ the current matter density very close to the critical density $\rho_{C}$, i.e. $\rho_{0} \sim \rho_{C} \sim 10^{-26} \mathrm{~kg} \cdot \mathrm{m}^{-3}$.

$T_{0}$ the current temperature of the cosmic microwave background (CMB) which is $2.7 \mathrm{~K}$, i.e. $T_{0} \sim 10^{0.43} \mathrm{~K}$.

Between $t_{0}$ and $t_{R E C}$ date of recombination, the Universe follows a dynamic dominated by matter.

The Universe undergoes a scale factor of the order of $a\left(t_{R E C}\right) / a\left(t_{0}\right) \sim 1 / 1100 \sim 10^{-3.04}$.

All of these previous data are measurements from observation and are well explained by theory. We will use this data to deduce the evolution of the Universe in the frame of our solution. Our only adjustment variable will be the scale factor. The other parameters are deduced using the previous formulas.

Figure 1 which follows makes it possible to synthesize the problem which one seeks to solve. We put there the chronology of the history of the Universe in these 3 major epochs (going back in time), dominated by matter, dominated by radiation, dominated by a cosmological constant $\Lambda$. The light blue backgrounds

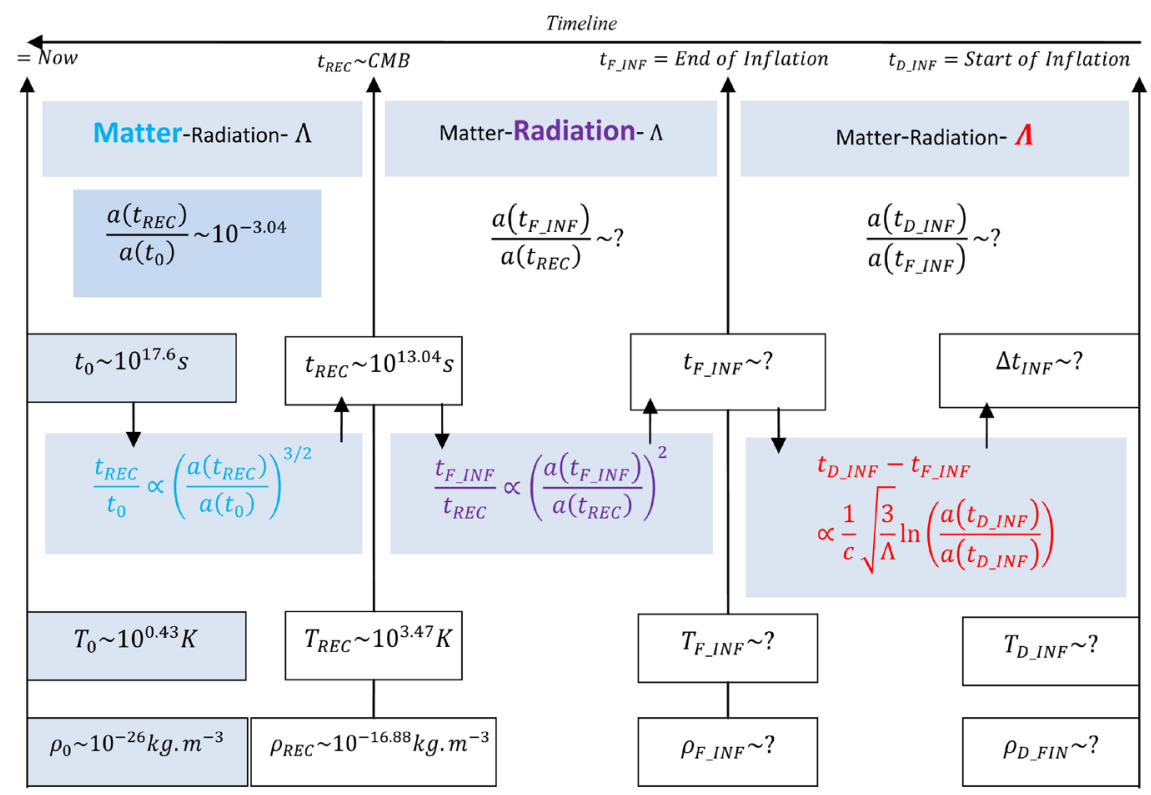

Figure 1. Sought values of parameters achieving inflation as expected by theory. 
are the information that theory and observation provide us and which are not modified by our solution and on which we will rely to define the other parameters indicated by a question mark. The values for recombination are indicated because our solution does not modify this epoch.

Our study will now be carried out in a succession of calculations which will make it possible to notice important facts or to reveal problems of coherence which we will raise step by step to tend towards a coherent solution which will answer all the expectations of the theory of inflation. As a bonus, we will no longer need the cosmological constant.

The time $t_{F_{-} I N F}$ corresponds to the end of inflation. In our solution, inflation is caused by the symmetry breaking giving rise to gravitation. The theory predicts that this symmetry breaking occurs before $10^{-43} \mathrm{~s}$. We will therefore have $t_{F_{I} I N F}<10^{-43} \mathrm{~s}$. We will do a first calculation with $t_{F_{I} I N F} \sim 10^{-43} \mathrm{~s}$. We will then do a second calculation with $t_{F I N F} \sim 10^{-44} \mathrm{~s}$. We will notice that between these two times the nature of the evolution of the Universe changes radically. We will then calculate the time which corresponds to this change in nature and for which the Universe generates inflation.

Let $t_{F_{I} I N F} \sim 10^{-43} \mathrm{~s}$ :

This time allows us to obtain the scale factor imposed by general relativity thanks to the relation:

$$
\frac{t_{F_{I} I N F}}{t_{R E C}} \propto\left(\frac{a\left(t_{F_{I N F}}\right)}{a\left(t_{R E C}\right)}\right)^{2}
$$

The calculation gives:

$$
\frac{a\left(t_{F_{-} I N F}\right)}{a\left(t_{\text {REC }}\right)} \sim\left(\frac{10^{-43}}{10^{13.04}}\right)^{1 / 2}=10^{-28.02}
$$

For the temperature, the equation (7) gives:

$$
T_{F_{-} I N F}=T_{R E C}\left(\frac{a\left(t_{F_{I} I N F}\right)}{a\left(t_{R E C}\right)}\right)^{-1} \sim 10^{3.47} \times 10^{28.02}=10^{31.49} \mathrm{~K}
$$

For the density of matter in a universe dominated by radiation, the relation (8) gives:

$$
\rho_{F_{-} I N F}=\rho_{\text {REC }}\left(\frac{a\left(t_{F_{-} I N F}\right)}{a\left(t_{R E C}\right)}\right)^{-4} \sim 10^{-16.88} \times 10^{112.08}=10^{95.2} \mathrm{~kg} \cdot \mathrm{m}^{-3}
$$

Now let's use the equations of our solution. To begin, we determine the mass (from thermal energy) of the particles resulting from the symmetry breaking giving birth to gravitation by the relation (5):

$$
M=\frac{3 k_{B} T}{c^{2}} \sim 3 \frac{10^{-22.86} \times 10^{31.49}}{10^{16.95}}=10^{-7.85} \mathrm{~kg}
$$

We then deduce the distance between particles by the relation (6): 


$$
d_{\text {intra }}=\left(\frac{M}{\rho_{F_{-} I N F}}\right)^{1 / 3} \sim\left(\frac{10^{-7.85}}{10^{95.2}}\right)^{1 / 3}=10^{-34.35} \mathrm{~m}
$$

Which gives us the pressure within the primordial cloud of particles (resulting from the gravitational force) by the relation (4):

$$
p_{\text {F_INF }} \sim-\frac{G M^{2}}{d_{\text {intra }}^{4}} \sim-\frac{10^{-10.18} \times 10^{-15.7}}{10^{-137.4}}=-10^{111.52}
$$

Let's do the second calculation at $t_{F_{-} I N F} \sim 10^{-44} \mathrm{~s}$ :

Thanks to the relation (10) the calculation gives:

$$
\frac{a\left(t_{F \_I N F}\right)}{a\left(t_{\text {REC }}\right)} \sim\left(\frac{10^{-44}}{10^{13.04}}\right)^{1 / 2}=10^{-28.52}
$$

For the temperature, the equation (7) gives:

$$
T_{F_{-} I N F}=T_{R E C}\left(\frac{a\left(t_{F_{-} I N F}\right)}{a\left(t_{R E C}\right)}\right)^{-1} \sim 10^{3.47} \times 10^{28.52}=10^{31.99} \mathrm{~K}
$$

For the density of matter in a universe dominated by radiation, the relation (8) gives:

$$
\rho_{F_{-} I N F}=\rho_{R E C}\left(\frac{a\left(t_{F_{-} I N F}\right)}{a\left(t_{R E C}\right)}\right)^{-4} \sim 10^{-16.88} \times 10^{114.08}=10^{97.2} \mathrm{~kg} \cdot \mathrm{m}^{-3}
$$

We determine the mass (from thermal energy) of the particles resulting from the symmetry breaking giving rise to gravitation by relation (5):

$$
M=\frac{3 k_{B} T}{c^{2}} \sim 3 \frac{10^{-22.86} \times 10^{31.99}}{10^{16.95}}=10^{-7.35} \mathrm{~kg}
$$

We then deduce the distance between particles by the relation (6):

$$
d_{\text {intra }}=\left(\frac{M}{\rho_{F \_I N F}}\right)^{1 / 3} \sim\left(\frac{10^{-7.35}}{10^{97.2}}\right)^{1 / 3}=10^{-34.85} \mathrm{~m}
$$

Which gives us the pressure within the primordial cloud of particles (resulting from the gravitational force) by the relation (4):

$$
p_{F_{-} I N F} \sim-\frac{G M^{2}}{d_{\text {intra }}^{4}} \sim-\frac{10^{-10.18} \times 10^{-14.7}}{10^{-139.4}}=-10^{114.52}
$$

Between these 2 times, we have an important physical change. Let's calculate the value of the expression $\rho c^{2}+3 p$.

$$
\begin{aligned}
& \text { For } t_{F_{-} I N F} \sim 10^{-43} \mathrm{~s} \\
& \qquad \rho_{F_{-} I N F} c^{2}+3 p_{F_{-} I N F}=10^{95.2} \times 10^{16.95}-10^{0.48} \times 10^{111.52}=10^{112.15}-10^{112}>0 \\
& \text { For } t_{F_{-} I N F} \sim 10^{-44} \mathrm{~S} \\
& \quad \rho_{F_{-} I N F} c^{2}+3 p_{F_{-} I N F}=10^{97.2} \times 10^{16.95}-10^{0.48} \times 10^{114.52}=10^{114.15}-10^{115}<0
\end{aligned}
$$

There is a time when this expression is zero and then becomes negative. And a condition for obtaining inflation is that this expression becomes negative, this is 
therefore the case with the gravitational energy of the primordial cloud of positive and negative heavy masses when it reaches a certain level of density. We can look for the changeover moment when the expression cancels. We find $t_{F_{-} I N F} \sim 10^{-43.16} \mathrm{~s}$ (for $\left.\frac{a\left(t_{F_{-} I N F}\right)}{a\left(t_{R E C}\right)} \sim 10^{-28.1}\right)$. At this stage, we already have two fabulous results, a primordial cloud of particles which has a negative pressure and which moreover makes it possible to verify a relation necessary for inflation. We will now see that this solution will also make it possible to eliminate the cosmological constant which is a parameter necessary for agreement with the observations but added in an "ad hoc" way in current theories.

\subsection{New Explanation of the Cosmological Constant}

As we will see a little later, the symmetry breaking seems to have all the qualities required to maintain itself for some time (the duration of inflation). In our solution, inflation is in fact the symmetry breaking. During this epoch the physical conditions will remain relatively constant (verifying the hypothesis known as "slow roll"). During this epoch the Universe evolves according to the model of De Sitter. He then checks the following equation:

$$
c^{2} \Lambda-4 \pi G\left(\rho+\frac{3 p}{c^{2}}\right)=\frac{3 \ddot{a}(t)}{a(t)}=c^{2} \Lambda+c^{2} \Lambda_{\rho}
$$

In our solution, we will take $\Lambda=0$, the cosmological constant will then be replaced by the values of the density of matter and the negative pressure of the primordial cloud which will be maintained during this epoch of inflation as we will see later $\left(\Lambda_{\rho_{ \pm}}=\frac{4 \pi G \rho_{I N F}}{c^{2}}\left(\frac{3\left|p_{I N F}\right|}{\rho_{I N F} c^{2}}-1\right)\right)$. This equation has the solution:

$$
\frac{a\left(t_{F_{-} I N F}\right)}{a\left(t_{D_{-} I N F}\right)} \propto \mathrm{e}^{\sqrt{-\frac{4 \pi G \rho_{I N F}}{3}\left(1+\frac{3 \rho_{I N F}}{\rho_{I N F} c^{2}}\right)}\left(t_{F_{I N F}}-t_{D_{I N F}}\right)}=\mathrm{e}^{\left.\sqrt{\frac{c^{2} \Lambda_{\rho_{ \pm}}}{3}\left(t_{F_{I N F}}\right.}-t_{D_{I N F}}\right)}
$$

This equation allows us to calculate the time that inflation can last in our solution. It will allow us to refine the previous solution so that we get inflation that corresponds to what is expected by solving two problems.

1 st problem the duration of inflation consistent with $t_{F_{-I N F}}$ :

If we look at what happens for $t_{F_{-} I N F} \sim 10^{-43.16} \mathrm{~s}$ (previous date for inflation to be possible), we realize by calculation (using the same procedures and equations than previously) that for a factor $\frac{a\left(t_{F_{I} I N F}\right)}{a\left(t_{D_{\perp} I N F}\right)}=10^{1}$ the duration of inflation is $t_{F_{-} I N F}-t_{D_{-} I N F} \sim 10^{-41.81} \mathrm{~s}$. This duration is greater than $t_{F_{I} I N F}$ which is not possible (unless you start the history of the Universe with a negative time). We must therefore have a duration less than or equal to $t_{F_{I N F}}$ for the solution to be consistent. Now by increasing the radiation epoch (which increases the density at time $t_{F_{I} I N F}$ and which decreases $t_{F_{I} I N F}$ ) we systematically arrive at consistent durations (i.e. less than $t_{F_{-} I N F}$ ). For example, one of the 1 st values 
which makes it possible to obtain a coherent solution with $\frac{a\left(t_{F_{-} I N F}\right)}{a\left(t_{D_{-} I N F}\right)}=10^{1}$ is $\frac{a\left(t_{R E C}\right)}{a\left(t_{F_{-} I N F}\right)} \sim 10^{28.66} \quad$ (which then gives us $t_{F_{-} I N F} \sim 10^{-44.28} \mathrm{~s}$ ) with an inflation duration of $t_{F_{-} I N F}-t_{D_{-} I N F} \sim 10^{-44.29} \mathrm{~s}$ (therefore less than $t_{F_{-} I N F}$ ).

On the other hand, by increasing beyond a certain threshold the scale factor during inflation, $\frac{a\left(t_{F_{-} I N F}\right)}{a\left(t_{D_{-} I N F}\right)}$, the duration of inflation becomes incoherent again (greater than $t_{F_{-} I N F}$ ). For example, for the previous case $t_{F_{-} I N F} \sim 10^{-44.28} \mathrm{~s}$, the solution becomes incoherent again from $\frac{a\left(t_{F_{-} I N F}\right)}{a\left(t_{D_{-} I N F}\right)}=10^{1.03}$. In other words, for times $t_{F_{-} I N F}<10^{-44.28} \mathrm{~S}$ we always manage to find a range of scale factor $\frac{a\left(t_{F_{-} I N F}\right)}{a\left(t_{D_{-} I N F}\right)}$ for which the duration inflation is possible, but this range always has a maximum value. We have therefore at this stage solved this 1st problem by having a whole set of possible values to make coherent our solution with inflation. But the duration of this inflation cannot be chosen as long as we want (because of this previous maximum value) which leads us to our $2^{\text {nd }}$ problem.

\section{$2^{\text {nd }}$ problem the factor of scale since the beginning of the Universe:}

For a certain number of modeling problems of our Universe to be solved (problem of flatness, of the horizon...), our Universe must have known a scale factor of $\frac{a\left(t_{0}\right)}{a\left(t_{D_{-} I N F}\right)} \sim 10^{60}$. Therefore, among the set of possible values (obtained in the solution of the 1st problem), we must verify that we are able to reach this criterion. Since this set of values has a maximum, we don't necessarily meet this criterion. For example if we take the previous case for $t_{F_{-} I N F} \sim 10^{-44.28} \mathrm{~s}$, we have $\frac{a\left(t_{0}\right)}{a\left(t_{R E C}\right)} \sim 10^{3.04}, \frac{a\left(t_{R E C}\right)}{a\left(t_{F_{-} I N F}\right)} \sim 10^{28.66}$ and $\frac{a\left(t_{F_{I} I N F}\right)}{a\left(t_{D_{-} I N F}\right)}=10^{1.03}$ at most, i.e. a total $\frac{a\left(t_{0}\right)}{a\left(t_{D \_I N F}\right)} \sim 10^{32.73} \ll 10^{60}$. Fortunately, the more the radiation epoch increases (and therefore also the density of matter at time $t_{F_{I I N F}}$ ), the higher the maximum possible scaling factor during inflation $\frac{a\left(t_{F_{-} I N F}\right)}{a\left(t_{D_{-} I N F}\right)}$ increases. So, by continuing to decrease $t_{F_{-} I N F}$ we get the ability to have increasing scale factor inflations. The first later value of $t_{F_{-} I N F}$ for which we can obtain the scale factor $\frac{a\left(t_{0}\right)}{a\left(t_{D \_I N F}\right)} \sim 10^{60}$ is $t_{F_{-} I N F} \sim 10^{-47.088} \mathrm{~s}$ (with $\frac{a\left(t_{R E C}\right)}{a\left(t_{F \_I N F}\right)} \sim 10^{30.064}$ ), it allows us to reach $\frac{a\left(t_{F_{I} I N F}\right)}{a\left(t_{D_{-} I N F}\right)}=10^{26.92}$ for a duration of $t_{F_{-} I N F}-t_{D_{-} I N F} \sim 10^{-47.088} \mathrm{~s}$. At this 
stage, we have mathematically found the solution which meets all the theoretical and observational expectations (i.e. a scale factor of $10^{60}$ and an inflation which has time to develop with the bonus of a zero cosmological constant).

In summary, Figure 2 presents the results of this solution.

\subsection{Comments on the Field of Validity of the Results Obtained}

Of course, these quantitative results are to be taken with great care. They are certainly very approximate because they are at the limits (and even slightly

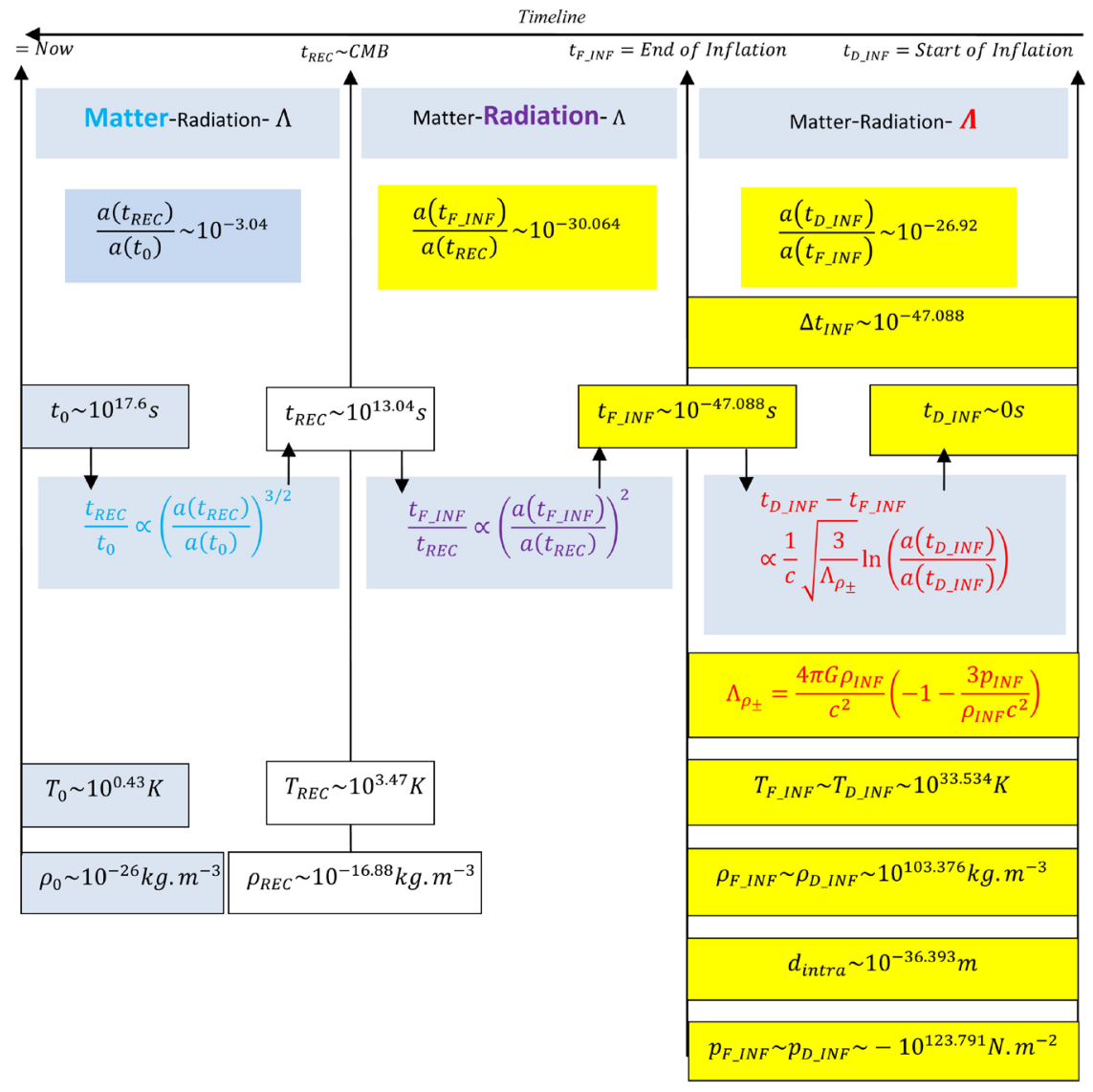

Figure 2. Values of parameters achieving inflation as expected by theory. Note: This solution, in addition to eliminating the cosmological constant and satisfying the expected scale factor, also has a remarkable and interesting characteristic. Inflation can a priori start as close as possible to the instant $t_{D_{-} I N F}=0$. Inflation being linked to the symmetry breaking, this characteristic would mean on the one hand that "physical" time (our history) would start with the creation of the first massive particles and on the other hand that this symmetry breaking would also start at $t_{D_{-} I N F} \sim 0$. If we want this duration to be shorter than $t_{F_{-} I N F}$ so as not to start so close to $t_{D_{-} I N F}=0$, this solution allows it, but this means that $t_{F_{-} I N F}$ must be further reduced. For example, at $t_{F_{-} I N F} \sim 10^{-49.06} \mathrm{~s}$ (with $\left.\frac{a\left(t_{R E C}\right)}{a\left(t_{F_{-} I N F}\right)} \sim 10^{31.05}\right)$, we can obtain a duration 10 times shorter, $t_{F_{-} I N F}-t_{D_{-} I N F} \sim 10^{-50.06} \mathrm{~s}$ with $\frac{a\left(t_{F_{I} I N F}\right)}{a\left(t_{D_{-} I N F}\right)}=10^{25.91}$ while having our scale factor of $10^{60}$. 
beyond) of what is considered to be the validity of gravitational theories. But the limit of validity of a theory can mean either significant deviations in quantitative terms or inability to integrate new phenomena in qualitative terms. For example, the Newtonian theory of gravitation can predict a curvature of light rays like general relativity does, but it obtains values with a great imprecision compared to general relativity. In this case, the Newtonian theory leaves its domain of validity more in quantitative than qualitative terms but it can predict a curvature (but with a wrong value). If general relativity did not exist, Newtonian theory would still provide us with important information, the reality of the phenomenon of the light deviation and an order of magnitude, even if it is a very approximate one. On the other hand, for gravitational waves, the Newtonian theory leaves its domain of validity in qualitative terms. It is unable to predict anything in this wave domain. If general relativity did not exist, Newtonian theory would give us no information. In our situation, in the absence of a quantum theory of gravitation, if we had not been able to obtain a solution to inflation within this theoretical framework, we would certainly have been in the latter case. But the fact of having succeeded in obtaining an extremely coherent solution can give us hope that we are rather in the first case of a confirmation of the phenomenon but with very approximate orders of magnitude, that a quantum theory of gravitation can correct in quantitative terms. In other words, even if we are very skeptical about the values obtained, we think that the astonishing coherence obtained should be taken into consideration.

When defining the pressure, we introduced a parameter $\varepsilon \leq 1$. To simplify the reasoning, we have so far taken $\varepsilon=1$. As announced, we give the results of our solution for $\varepsilon=1 / 4$. We can see that our solution is stable, the computation always consistent and with the same orders of magnitude. We could safely take even smaller values of $\varepsilon$. The results are slightly modified but as we have just specified, without a quantum theory of gravitation, it would be futile to obtain precise values. For $\varepsilon=1 / 4$, we get:

$$
\begin{aligned}
& \frac{a\left(t_{F_{-} I N F}\right)}{a\left(t_{R E C}\right)} \sim 10^{-30.36} ; \frac{a\left(t_{D_{-} I N F}\right)}{a\left(t_{F_{-} I N F}\right)}=10^{-26.61} ; t_{F_{-} I N F} \sim \Delta t_{I N F} \sim 10^{-47.68} ; \\
& T_{F_{-} I N F} \sim T_{D_{-} I N F} \sim 10^{33.83} ; \rho_{F_{-} I N F} \sim \rho_{D_{-} I N F} \sim 10^{104.56} ; \\
& d_{\text {intra }} \sim 10^{-36.69} ; p_{F_{-} I N F} \sim p_{D_{-} I N F} \sim-10^{124.965}
\end{aligned}
$$

We will now be able to rely on this mathematically consistent solution to obtain yet other very interesting results which will show the physical consistency of this solution.

\subsection{Maintaining the Symmetry Breaking (i.e. Inflation)}

We have just shown that our solution of negative gravitational mass makes it possible to solve mathematically the 1st instants of the Universe. It remains to show that this solution is also physically feasible by showing the only assumption (in addition to the existence of negative heavy mass) that we used: the capacity 
of the symmetry breaking to be maintained.

In our solution, we have a primordial cloud, created by the symmetry breaking, of very high density and very high repulsive pressure within each pair. Physics in this epoch of inflation will therefore be exclusively controlled by the countless collisions of particles generated by this internal force. Indeed, beyond each pair, the gravitational mass of the cloud appears to be zero (screening effect), only the huge gravitational impulse at the creation of the pairs of positive and negative particles dominates (in a first time). In addition, with this impulse associated with the enormous density, the average distance between two collisions is short enough not to be influenced by other forces.

The symmetry breaking begins when the temperature and the pressure reach a precise critical value (as for a phase transition). One might think then that the creation of the particles causes a drop-in temperature and the repulsion within the pairs a drop-in pressure (by decreasing the density). But two phenomena counteract these declines.

Density maintained in the heart of the primordial cloud by its extent.

The internal force which increases the distance between the two particles of a pair generates a "void to be filled" and the two particles are projected in the neighboring pairs. But the neighboring pairs undergo the same effect. Therefore, within an extended cloud, the "void to be filled" is filled by neighboring particles maintaining a high density. It is first on the edges of the cloud that the effect of decreasing the density (and therefore the pressure) is felt (by the strong decrease in the neighborhood which cannot compensate for the "void to be filled"). And like a retrograde shock wave this depression will propagate from the edges towards the heart of the cloud. For a certain duration, depending on the size of this primordial cloud, its heart will maintain a relatively constant pressure until the wave of depression arrives.

Temperature maintained by countless collisions:

With countless collisions, a very large part of the kinetic energy will be transformed into thermal energy. This will result in maintaining a relatively constant temperature within the cloud. And as for pressure, it is first of all the edges of our primordial cloud which will undergo a drop-in temperature because there will be fewer collisions (the density decreases there faster) but the heart will experience for a certain duration relatively constant temperature.

Maintaining the symmetry breaking leading to the maintenance of inflation:

This maintenance of pressure and temperature conditions makes it possible to satisfy the hypothesis traditionally required by theories of inflation known as "slow roll". Our solution thus validates the mathematical hypotheses of our previous calculation. But it also allows us to physically understand what is going on. This maintenance of the physical conditions around the critical point of symmetry breaking engenders the maintenance of the creation of pairs of particles which makes it possible to maintain inflation, because only the creation of the pairs of particles is the source of our inflation. As soon as the creation of pairs stops, inflation almost immediately turns into an expansion that decelerates 
(notably due to collisions but also simply by the absence of "accelerating energy"). It can be noted that this mechanism also has the advantage of maintaining the same physical conditions on the whole cloud around the same values (those of the critical point) on large scales, which will certainly help to lead later to the very large homogeneity of the $\mathrm{CMB}$.

Let us translate graphically (Figure 3 ) into $1 \mathrm{D}$, the effect on inflation of this mechanism of maintenance. We assume (as we have done before) that $d_{\text {inter }} \sim d_{\text {intra }}$ and therefore that the particles move from $d_{\text {intra }}$ on average between two collisions. For simplicity, we have not considered the neighboring pairs which help to support the pressure, but only the created pairs.

We took a factor of 3 for the expansion because it creates the necessary and sufficient space to create a "void to be filled" of the size of a pair (which maintains the density and therefore checks the hypothesis of "slow roll"). We note in this mechanism the direct proximity to the $\mathrm{N}$-folding parameter (which gives it an interesting physical meaning). Indeed, we find the sixty $\mathrm{N}$-folding expected during inflation because we previously obtained a scale factor of $10^{26.92}$ which represents $10^{26.92} \sim 3^{56}$ i.e. $56 \mathrm{~N}$-folding with this factor 3 . And as the distance between pairs $d_{\text {inter }}$ checks $d_{\text {inter }} \sim d_{\text {intra }}$, we can interpret this distance $d_{\text {intra }}$

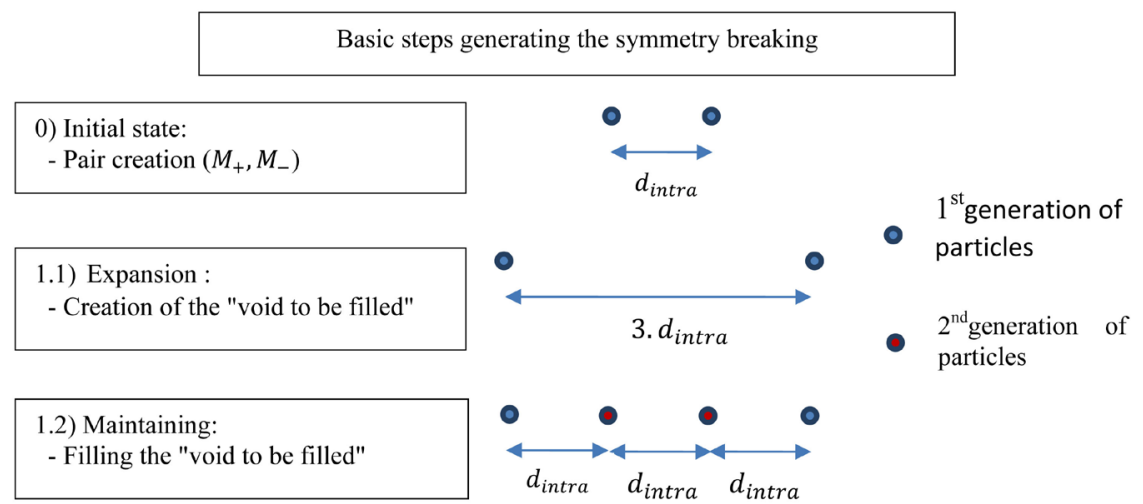

Evolution of inflation when maintaining the symmetry breaking

$$
\text { 2.1) } 2^{\text {nd }} \text { Expansion: }
$$

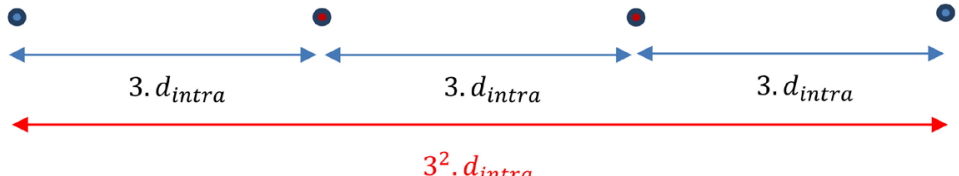

2.2) $2^{\text {nd }}$ Maintaining:

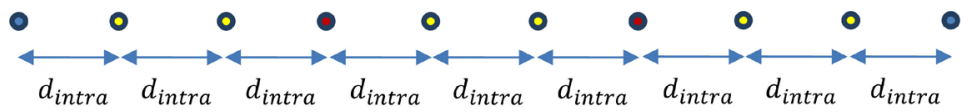

Figure 3. Simplified representation in 1D of the effect of maintaining the symmetry breaking on inflation. 
as the distance between 2 collisions. Therefore, an N-folding can define a measure of time in terms of collision. Thus, the duration of our inflation (56 $\mathrm{N}$-folding) can be interpreted as the time of 56 collisions. This measurement of time will give us a way of comparison for the transitional phase following inflation.

Note: The adequate functioning of this mechanism which satisfies our need to maintain the symmetry breaking (by this factor 3) makes it possible to justify posteriori the hypothesis $d_{\text {inter }} \sim d_{\text {intra }}$ taken in all the preceding calculations.

\section{From Inflation to the Radiation Epoch}

We have obtained many results which show the great coherence of this solution (date of inflation in agreement with the symmetry breaking expected for the appearance of gravitation, a matter verifying $\rho c^{2}+3 p<0$ rendering the cosmological constant useless, the possibility of reaching the desired scale factor). This solution also makes it possible to physically explain the appearance of a radiation epoch (which we have mathematically postulated so far) which follows the inflation phase. Indeed, as we said earlier, the dynamics of this primordial cloud will evolve according to a physics governed by countless random shocks and a negligible gravitational interaction (even if the huge initial impulse is gravitational), due to a distribution very fine and very dense of positive and negative masses (without lumps and with screening effect). We then have four possible kinds of collisions with two particles, according to the sign of the heavy mass of each particle: $(+,+)(-,-)(+,-)(-,+)$. We will only consider collisions with 2 particles. A priori, the higher the density of particles, the more the probability of collision with more than two particles increases, but we can still expect that the majority of collisions will be with 2 particles. In this theoretical framework we then expect that, at the end of the 1st collision on all the particles (equiprobability of shocks), we have:

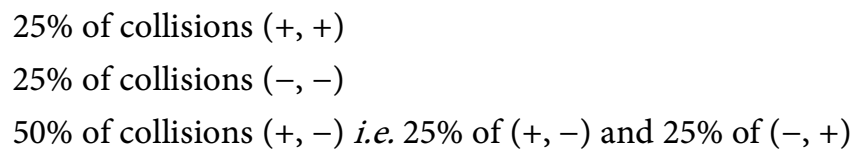

This statistic allows us to predict the birth of a radiation and reheating phase after inflation. As demonstrated in [2], if negative heavy masses exist, they must be carried by antiparticles. So, even if at this stage the electric charge has not yet emerged, the pair of particles $(+,-)$ resulting from the symmetry breaking is therefore a particle-antiparticle pair. Collisions $(+,-)$ will therefore generate photons. Let's take a look at how photon-particle ratios change during and after inflation.

\subsection{Inflation Dominated by Matter}

During the phase of maintaining inflation, if the 2 particles within the exploding pair do not annihilate themselves with their neighbor, there will be creation of 1 pair of particles (by symmetry breaking at the critical point) which will fill the 
"void to be filled" and maintain density (as in Figure 3). If, however, one of them annihilates, 2 pairs will be created to compensate for the double "void to be filled", and if the 2 annihilate, 3 pairs will be created to compensate for the triple "void to be filled". In all these situations, if annihilation creates only 1 photon, there is more creation of particles than of photons. Same if the annihilation only creates 2 photons because we will only have $50 \%$ annihilation. In other words, during this epoch of inflation it is reasonable to think that matter dominates. However, we can also expect that this photon creation process participates in the slow sliding of the physical conditions around the critical point. It could be the main element which comes to stop the maintenance of the symmetry breaking and put an end to the hypothesis of "slow roll".

\subsection{Post Inflation Dominated by Radiation}

On the other hand, when inflation stops (i.e. the creation of particles), we can understand that the multitude of collisions will change the Universe into an epoch dominated by photons (radiation epoch). We can also try a rough calculation to give us an idea of the "duration" (in terms of number of collisions) of the transition to this radiation phase. According to previous statistics, after the 1 stcollision, we already have $50 \%$ of the particles that have been transformed into radiation. We know on the one hand that the current radiation is dominated by the number of photons of the $\mathrm{CMB}$ and the observations give a density (at the present time) $\rho_{r, 0} \sim 4 \times 10^{8} \mathrm{~m}^{-3}$ [5]. On the other hand, the measurements (translated into proton terms) give for the baryonic matter

$\rho_{m, 0} \sim 5.5$ protons $\mathrm{m}^{-3}$ [5]. We can translate this value into the number of quarks (which are certainly the particles resulting from this 1stsymmetry breaking) even if this transformation does not change the calculated order of magnitude. Knowing that 1 proton is composed of 3 quarks, this gives

$\rho_{m, 0} \sim 16.5$ quarks $\mathrm{m}^{-3}$. The number of photons $N_{r}$ on the number of quarks $N_{m}$ at the time of the CMB thus gives withz the redshift (one takes account only of the comoving volume and not of the spectral shift for radiation because it does not change the number of photons explaining the power 3 and not 4):

$$
\frac{N_{r}}{N_{m}}=\frac{\rho_{r, 0} \cdot(1+z)^{3}}{\rho_{m, 0} \cdot(1+z)^{3}}=\frac{\rho_{r, 0}}{\rho_{m, 0}} \sim 10^{7.4}
$$

So, in terms of number of particles, the ratio doesn't depend on $z$. If we assume that this ratio can change very little during the radiation epoch, we must expect, at the end of this transition between inflation and radiation phase, to have a ratio between the number of photon and particle of heavy masses of the order of $10^{7.4}$

With our statistics, after " $i$ " collisions, the initial number of baryons $N_{m 0}$ decreases by half:

$$
N_{m i}=2^{-i} N_{m 0}
$$

At the same time, if $n_{v}$ corresponds to the number of photons produced by 
an annihilation $(+,-)$, the number of photons $N_{r i}$ becomes:

$$
N_{r i}=2^{-1}\left(N_{m 0}-N_{m i}\right) n_{v}=2^{-1} n_{v} N_{m 0}\left(1-\frac{N_{m i}}{N_{m 0}}\right)=2^{-1} n_{v} N_{m 0}\left(1-2^{-i}\right)
$$

And then:

$$
\frac{N_{r i}}{N_{m i}}=2^{i-1} n_{v}\left(1-2^{-i}\right) \underset{i \gg 1}{\longrightarrow} 2^{i-1} n_{v}
$$

After $p$ collisions (for $p>10$ ), we have $\frac{N_{r}}{N_{m}} \sim n_{v} 2^{p-1}$. For an annihilation generating $n_{v}=1$ photon, we need $p=7.4 \frac{\ln 10}{\ln 2}+1 \sim 26$ collisions. For an annihilation generating 10 photons, 22 collisions would be required. The order of magnitude is very stable with respect to this parameter and the same is true if we quadruple the number of baryons. In other words, a good twenty collisions at most in the random shock regime is enough to obtain the current proportions of our Universe. This is less than half the time of inflation. A short transition then leads from inflation to radiation epoch with a change in the nature of the collisions which must statistically generate fewer annihilations (or by a mechanism which compensates for them).

The rest of our study will now be more qualitative than quantitative and will focus on different characteristics that can be predicted in the frame of this solution.

\subsection{After Inflation, an Epoch of Reheating}

In addition to the collisions $(+,-)$ which produce the radiation phase, we have as many collisions $(+,+)$ and $(-,-)$ which occur. During these shocks, the kinetic energy is transformed into thermal energy. But while, during inflation, the symmetry breaking caused a transition which lowered the temperature that these shocks compensated for, the end of inflation (i.e. the symmetry breaking) necessarily led to an accumulation of thermal energy in particular during the good twenty collisions of the transition seen previously between the end of inflation and the radiation epoch. In parallel with this reheating, the infernal succession of hyper-energetic shocks of the particles which move away from each other (and for which the gravitational interaction can still be neglected) will lead to a braking of the expansion until a rhythm expected by theory for the radiation epoch. The energy of the expansion is thus partly recycled into thermal energy.

\section{Resumption of Gravitation}

\subsection{Formation of Universes and Their CMB}

Whereas until now the enormous primordial impulse, the fine gravitational neutrality (screening effect beyond the pair) and the hyper density of particles imposed a dynamic of random shocks for this primordial cloud, the expansion will allow the particles to have a longer trajectory time between 2 collisions and 
to be deviated more appreciably by the attraction and repulsion of neighboring particles. We are now entering an era where gravitation gradually regains control with its force of attraction and repulsion which sees its radius of action gradually increase. The screening effect was previously applied just beyond the 1 stpair and gradually it will be applied beyond the few neighboring pairs $\left(2^{\text {nd }}\right.$, $3^{\text {rd }}, \ldots$ etc. ...). The average trajectory of particles between 2 collisions is gradually increasing. However, the situation is not symmetrical between attraction and repulsion because expansion, even if it is no longer inflationary, is still high in comparison to the gravitational interaction. We have a gravitation which increases but remains weak and an expansion which decelerates but remains high. The particles therefore continue to move away but the particles which attract move away a little less and those which repel move away a little more. Concretely the positive heavy masses will tend to move less away from each other, so will the negative masses. And at the same time, the positive and negative masses will move away more effectively. There will be a more marked effect of separation of the masses of opposite signs than of regrouping of the masses of the same signs (no lumps but regular homogenization). There will therefore begin to be a segregation of the heavy masses according to their sign. Slowly, gravitation will increase (the radius of the screening effect will increase) in these areas of homogeneous masses (purely positive or purely negative). In these zones the gravitation will slow down the expansion and between these zones the expansion and the repulsion will gradually separate the zones of homogeneous masses in an increasingly marked way. The characteristic size of these homogeneous zones (and their regularity) will depend on the intensity of the gravitation. The weaker the gravitation, the more the homogeneous zones will be extended and regular (an important attraction would generate a strong density gradient and therefore a more irregular zone in density). This is what happens at the beginning of the radiation epoch when a weak effect of gravitation begins to be felt and it is at this time that the first large homogeneous structures must form which will give birth to future universes including ours [3]. The shocks, even if they have decreased, are still numerous enough to also contribute to maintaining the regularity (in temperature and density) of the homogeneous zones. We can also note that the extreme initial regularity of the primordial cloud (regularity on the scale of the pair of particles, i.e. at a distance of $d_{\text {intra }}$ over the whole of the primordial cloud) gives initial conditions which favor obtaining very large homogeneous and regular zones (the gravitational effects described previously applying identically over the whole cloud, invariant by translation). We will notice the scale at which we are at this stage of our study, we are talking about several universes, some of positive heavy masses like ours and others of negative heavy masses (our $1^{\text {st }}$ neighbors).

From Figure 4, we can say that, under the mutual influence of attractive, repulsive gravitation and expansion, we have a kind of phase transition which takes us from the primordial cloud (heterogeneous on the scale of the pair of particles and homogeneous beyond the pair) to the creation of a set of universes 


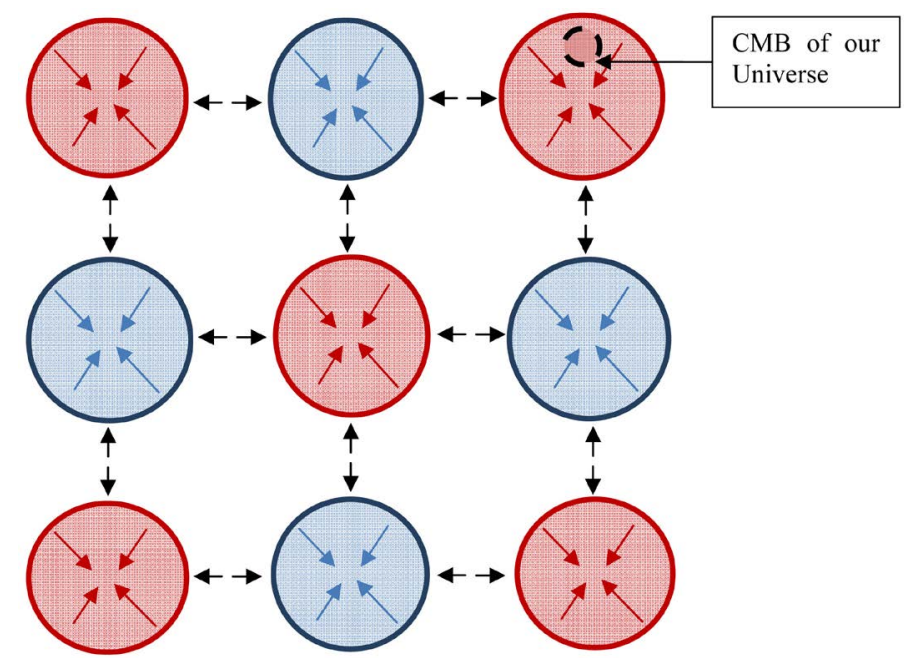

Figure 4. Simplified representation of the establishment of a universe structure (cf. [3]) whose neighboring universes are of opposite heavy masses. The arrows represent the sense of gravitation, attractive within universes or anti-universes, repellent between universes and anti-universes.

(homogeneous on the scale of a universe and heterogeneous beyond a universe). This last state would be that observed in the CMB, where the segregation of the universes would be achieved (heterogeneity pushed beyond the universes and therefore beyond our Universe and the $\mathrm{CMB}$ ) but the homogeneity within the universes would still be preserved (regularity of the $\mathrm{CMB}$ ).

\subsection{Digressions That Go beyond CMB}

And if we describe the physical conditions that prevail for this "phase transition" (i.e. the intensities of attraction, repulsion and expansion), we can then imagine the evolution of these conditions in the time, which leads us to potentially define 3 regimes of combinations of gravitational interaction and expansion. These 3 regimes could be the structuring elements leading first to the universes, then to the filaments (or walls) and finally to the superclusters. These 3 structures would finally be the most influenced by antimatter (negative heavy masses). The other structures (clusters, galaxies, etc.) would be under the influence of the attractive gravitation of its own universe. Let us briefly describe these 3 regimes (early, intermediate and late), remembering that we are in an epoch where the shocks have diminished (in any case it no longer dominates the dynamics of the primordial cloud):

Early epoch (\{“attraction $<<$ repulsion" $\}+$ expansion) generating the universes:

At the beginning locally (on the scale of a few particles) there is little gravitational attraction and it is erased by expansion. There is more gravitational repulsion which is accentuated by the expansion. These two effects with this intensity ratio make it possible to locally separate the masses of opposite signs while keeping the masses of the same sign in a regular arrangement but which contin- 
ues to expand. The density of masses of the same sign will therefore continue to decrease while slowing down but above all in a very smooth manner without lumps (no density gradient). We could speak of local soft segregation.

On a large scale, the gravitation of these homogeneous growing zones is neutralized by the nullity of the sum of the heavy masses. But the translational invariance of local soft segregation (due to the homogeneity of the primordial cloud) generates a large-scale increase in homogeneous areas. The image of the pair of initial particles replicates but on a larger scale, i.e. each particle, figuratively speaking, becomes a set of 2 particles of the same sign then $4,8, \ldots$ (the pair then becoming a quadruplet, octuplet, ...) and in a way at the time of the CMB (cf. Figure 4) the pair of particles has become a pair of universes (2 sets of $10^{80}$ particles).

There are thus homogeneous zones which effectively exclude the opposite masses within them while maintaining their regularity. And these areas also repel each other more and more effectively as they get bigger. This epoch should last a little beyond the CMB.

Remarks: The densities of inert masses (always positive) whether global or filtered according to the sign of the heavy mass always decrease at this stage because expansion dominates in the 2 registers, attractive and repulsive. On the other hand, the density of heavy mass, which were initially and globally zero beyond the 1stpair, remains null on the condition of taking increasingly large zones (including at least pairs of homogeneous zones of opposite heavy masses). But the densities of heavy masses in the homogeneous zones take a non-zero value which increases regularly (satisfying the regularity expected in the CMB) to reach the observed density of the CMB. One last non-trivial remark concerning these densities. In the homogeneous zones in heavy masses, the principle of equality of the heavy and gravitational masses is verified, in the heterogeneous zones this is not the case (cf. [2]).

Intermediate epoch (\{“attraction repulsion” $\}+$ expansion) generating the filaments, walls and voids:

With the creation of these large homogeneous zones, we moved from the image of a particle to that of a universe (a universe particle). We therefore gained a local scale with the creation of an internal structure in this universe particle. The influence of neighboring universe particles will now have consequences within the internal structures of these universe particles. We are now beyond the time of the CMB.

Little by little, locally (i.e. within a universe) the attraction (due to the masses within the same universe) and the repulsion (due to neighboring universes) become similar in intensity but the expansion which slows down is always present to accentuate the repellent effect. Matter densifies less quickly by attraction than repulsion dissociates it. The influence of neighboring opposite universes would then generate large voids while filaments and walls of matter would clump together.

Late epoch (\{“attraction >> repulsion”\} + expansion) generating superclusters: 
A little later locally (in homogeneous mass areas i.e. in the filaments of the universes), the attraction dominates and the repulsion weakens in these regions. The moderate expansion has less influence on gravitation and the filaments could start to fragment forming the superclusters by following the "over-densities" which appeared during the agglutination of matter along these "1D structures".

In this logic, as we said earlier, for these 3 structures, the influence of antimatter would finally be important. The other structures at lower scales (clusters, galaxies, etc.) would be relatively isolated from the repellent effects of neighboring anti-universes.

\section{Evolution of Expansion within the Universes}

The damping of the expansion could also be the first signs of stopping the increase in the distance between neighboring anti-universes which could start to slightly approach, giving birth to an accelerating compression of our Universe (as it seems have had one a few billion years ago).

Without the resumption of gravitation, expansion would continue without decelerating or accelerating (except by heat dissipation). But with gravitation, we have two behaviors, attractive and repulsive. Attractive gravitation tends to decrease the distances between particles and therefore to increase the attractive behavior. Repulsive gravitation, on the contrary, has the effect of increasing the distances between particles and therefore of reducing the repulsive behavior. The attraction is a phenomenon which is amplified while the repulsion tends to disappear locally. The repulsive gravitation which increases distances can only survive in the long term by shaping the largest heterogeneous structures (the "universes cluster") because it applies to scales larger than the attractive gravitation which will in turn shape the largest homogeneous structures within universes (clusters, galaxies... as already said previously, the geometrical transition between these 2 scales causing the creation of filaments, walls and superclusters).

But these two kinds of universes can cause two kinds of braking due to attraction (attraction within a universe and attraction between two universes of heavy masses of the same sign), the second one being able to cause an acceleration. An immediate braking locally (within the universes) and then a later braking on a larger scale (between the universes). But this second braking if it occurs between the universes of masses of the same sign implies that the universes of opposite signs which is between them undergo inversely a compression which will tend to accelerate them in planes perpendicular to the attraction of the neighboring anti-universes (Figure 4). The universes could undergo a succession of compressions and dilations which are damped over time [3].

\section{Evolution of the Geometry of the Primordial Cloud until the Filament of the Universes}

Here we will again approach the evolution of the primordial cloud but from a 
slightly different point of view. Previously, we rather placed ourselves within a universe (in particular ours but the story should be the same in all universes). This time, we will have a more global and external look at the universes to see the shape of the cloud as a whole. We will find some common elements seen previously.

\subsection{D-Ification of $1^{\text {st }}$ Kind - $1^{\text {st }}$ Volume Flattening of the Initial Ball in a Bubble}

At the end of inflation (when the cloud no longer creates particles), the primordial cloud will expand and for a non-null duration, which will depend on the size of this cloud, its heart will be crossed by particles coming from all the cloud. But after this duration, the particles will all have traveled a distance long enough to move away from the central area. The heart of this primordial cloud will then empty of all particles. The cloud will form a hollow ball and over time all of the matter will be on the "surface" of a ball like a balloon that is inflated (or like a shock wave which dissipates). The structuring of universes with homogeneous mass takes place in the thickness of this "surface". At this stage, it is not our Universe which forms this surface but all the universes which come to compose this surface (and which therefore undergo this flattening of their geometry).

\subsection{D-Ification of $2^{\text {nd }}$ Kind - 2nd Volume Flattening of the Universe Balls on the Surface of the Initial Bubble}

During the radiation epoch, the universes will then structure themselves on the surface of this $1^{\text {st }}$ bubble. The universes (with homogeneous mass) will then gradually influence each other. As explained in [3], these universes will form an alternating network of universes of positive and negative mass. Each universe will be surrounded by anti-universes (Figure 4). Each universe is expanding, the edges will be repelled by their neighbors. The edges of the universe balls will therefore undergo a slower expansion than their core. This will densify the edges. Again (but this time on the scale of each universe) the universe balls will become universe bubbles in which the matter will be concentrated at the edges. It is at this stage that we join the traditional image of our Universe on the surface of an inflating balloon. But in our solution, these universe bubbles are themselves on the surface of a "super bubble" from the primordial cloud.

\subsection{D-Ification-Flattening of the Surfaces of Universe Bubbles in Wire Elements}

The previous influence of neighboring universes (in the densification of edges) corresponded to an influence in the direction perpendicular to the surface. This gradually slowed their expansion in the direction perpendicular to the surface, favoring in the same time expansion in the plane of the edges. This influence of the anti-universes in the "plane" of the surface of the universe bubbles would make matter flee "towards the sides". In this case, their repelling effect should generate large "empty" zones and concentrate the matter along filaments on the 
surface of the universe bubbles. An image that helps to visualize this phenomenon could be that of a dense foam where the bubbles compressed against each other (equivalent to our gravitational repulsion) form denser lines at the interface of 3 bubbles.

\section{Conclusions}

The only hypothesis on which our study is based is the existence of negative heavy masses (with inert masses always positive). Unlike the authors who have dealt with the problem of negative mass, in our context 2 negative masses attract each other (like 2 positive masses between them) and 1 negative mass and 1 positive mass repel each other. This hypothesis is being tested and the first results should be known in less than two years. In this article, we show many problems that it is capable of solving or explaining in an extremely natural way in the cosmological context.

It makes inflation necessary by making the cosmological constant unnecessary and by following (and explaining) the traditional "slow roll" hypothesis. No dark energy is therefore necessary. It places this inflation at the onset of gravitational interaction (earlier than traditionally expected). More concretely, inflation results in a symmetry breaking (giving rise to the gravitational interaction) which is maintained over time.

It explains the epoch of radiation and reheating that follows. Indeed, at the end of inflation, the dynamics of the primordial cloud dominated by collisions, whose effects depend on the sign of the heavy masses of each colliding particle, explains the birth of radiation epoch by collisions $(+,-)$ and reheating epoch by collisions $(+,+)$ and $(-,-)$.

Then, the gradual resumption of gravitation is disturbed by the expansion favoring repulsion for a time and avoiding the agglutination of matter. The shocks decrease and become more elastic. Large-scale mass segregation takes place, giving rise to many universes that are homogeneous in masses and regular in matter density and temperature, making it possible to explain the characteristics of CMB.

One can even imagine that, with the mutual influence of increasing attractive gravitation on "small scale" and repulsive gravitation on "large scale", large structures (filaments and superclusters) are put in place. These structures would constitute the smallest scales influenced directly (for filaments and walls) and indirectly (for supercluster) by the antimatter of neighboring anti-universes. Let us also note in passing that, even if general relativity makes it possible to provide a mathematical framework describing the dynamics of the expansion of the Universe, it does not provide an explanation of the source of this expansion. This solution provides this explanation. Furthermore, this solution leads to a dizzying vision of a multitude of universes of positive masses (like our Universe) and negative masses (anti-universes of antiparticles).

Finally, it is remarkable (and certainly not a hazard) that at present the two 
concepts of negative heavy mass (consistent with theories of gravitation) and the 2nd component of gravitation ("gravitic" field at the origin of the Lense-Thirring effect) are two totally untapped or neglected sources of energy and at the same time that dark energy and dark matter are two unknown sources of energy. This study and [6] demonstrate that we can link these terms (on one hand dark energy and matter of negative heavy masses, on the other hand "gravitic" field of clusters and dark matter) and thus solve these two major problems of modern cosmology.

\section{Conflicts of Interest}

The author declares no conflicts of interest regarding the publication of this paper.

\section{References}

[1] Benoît-Lévy, A., et al. (2012) Introducing the Dirac-Milne Universe. Astronomy \& Astrophysics, 537, A78. http://arxiv.org/pdf/1110.3054.pdf https://doi.org/10.1051/0004-6361/201016103

[2] Le Corre, S. (2018) About the Negative Gravitational Mass. Open Access Library Journal, 5, 1-20. https://doi.org/10.4236/oalib.1104312

[3] Le Corre, S. (2015) Dark Energy, A New Proof of the Predictive Power of General Relativity. https://hal-ens-lyon.archives-ouvertes.fr/ensl-01122689

[4] Linde, A. (1990) Particle Physics and Inflationary Cosmology. https://arxiv.org/abs/hep-th/0503203 https://doi.org/10.1201/b16971

[5] Hobson, M., et al. (2006) General Relativity. https://doi.org/10.1017/CBO9780511790904

[6] Le Corre, S. (2015) Dark Matter, A New Proof of the Predictive Power of General Relativity. https://arxiv.org/abs/1503.07440 